\title{
Longitudinal emittance measurements at REX-ISOLDE
}

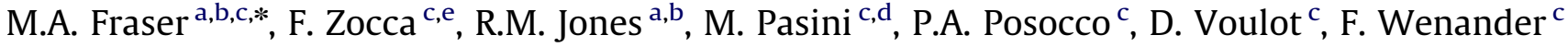 \\ ${ }^{a}$ Cockcroft Institute, Daresbury, UK \\ ${ }^{\mathrm{b}}$ University of Manchester, Manchester, UK \\ c CERN, Geneva, Switzerland \\ d Instituut voor Kern-en Stralingsfysica, Leuven, Belgium \\ e Instituto de Estructura de la Materia - CSIC, Madrid, Spain
}

\section{A R T I C L E I N F O}

\section{Article history:}

Received 8 August 2011

Received in revised form 20 September 2011

Accepted 12 October 2011

Available online 20 October 2011

\section{Keywords:}

Longitudinal emittance

Three-gradient measurement

Multi-gap bunching cavity

Silicon detector

\begin{abstract}
A B S T R A C T
We report on measurements of the longitudinal emittance at the Radioactive ion beam EXperiment (REX) at ISOLDE, CERN. The rms longitudinal emittance was measured as $0.34 \pm 0.08 \pi \mathrm{ns} \mathrm{keV} / u$ at the output of the RFQ and as $0.36 \pm 0.04 \pi \mathrm{ns} \mathrm{keV/u}$ in front of the third 7-gap split-ring resonator (7G3) using the three-gradient technique; systematic errors are not included but are estimated at approximately $10 \%$. The $86 \%$ emittance was measured a factor of approximately 4.4 times larger than the rms emittance at $1.48 \pm 0.2$ and $1.55 \pm 0.12 \pi \mathrm{ns} \mathrm{keV} / u$ at the RFQ and 7G3, respectively. The REX switchyard magnet was used as a spectrometer to analyse the energy spread of the beam as it was manipulated by varying the voltage of the rebuncher (ReB) and 7G3 cavities operating at nonaccelerating phases. The transfer matrix for a multi-gap bunching cavity is derived and suitably truncated to allow for the accurate reconstruction of the beam parameters from measurement. The technique for measuring the energy spread was rigorously simulated and validated. A silicon detector, in its development phase, was also exploited to measure the longitudinal beam properties. The measured longitudinal emittance is compatible with the acceptance of the HIE-ISOLDE superconducting linac upgrade.
\end{abstract}

\section{Introduction}

A campaign of emittance measurements was undertaken in order to better understand the quality of beams delivered by the Radioactive ion beam EXperiment (REX) at ISOLDE and to provide crucial information on the performance of the machine for ensuring its compatibility with the High Intensity and Energy (HIE) linac upgrade. The REX linac is shown next to the proposed HIE upgrade in Fig. 1 and included are the acronyms used for each accelerating structure. The upgrade will be staged with the high energy section arriving before the low energy section, thus it was important to investigate the beam properties in the REX linac at both low and high energies. For full details on the existing linac the reader is referred to [1] and to [2] for the HIE upgrade. During various shutdown periods in 2006 and 2008 the transverse emittance was measured with a dedicated emittance rig [3]. During 2010 and 2011 the longitudinal emittance was measured for the first time at opportune moments during pauses in the nuclear physics programme.

The experimental procedure and formalism for reconstructing the longitudinal beam parameters will be described before the results are presented and compared with simulation. In conclusion its results are summarised and put into perspective with the longitudinal acceptance of the superconducting upgrade.

\section{Experimental overview}

The longitudinal emittance was measured using the three-gradient technique before and after the IHS at $0.3 \mathrm{MeV} / u$ and $1.92 \mathrm{MeV} / u$. The three-gradient technique uses an rf device operating at non-accelerating phases to vary the energy spread of the beam as a function of the rf voltage. The response of the energy spread can then be measured and the emittance extracted by fitting the data using the Courant-Snyder formalism if the transfer matrix of the rf device is known [4]. The emittance could not be measured using the 9-gap IH

\footnotetext{
* Corresponding author at: University of Manchester, Manchester, UK.

E-mail address: mfraser@cern.ch (M.A. Fraser).
} 


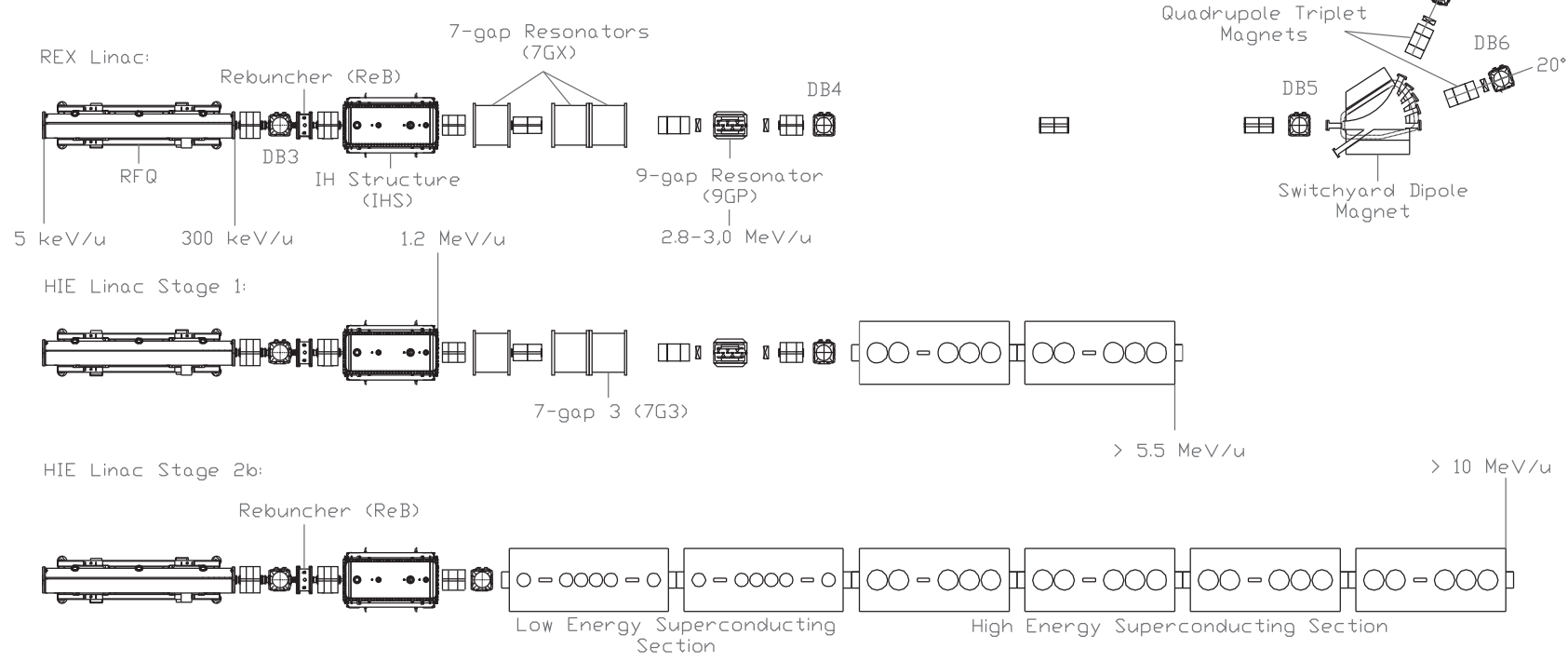

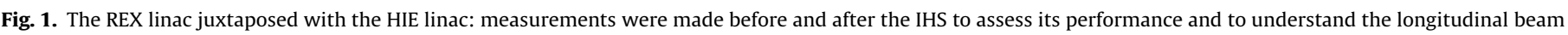
parameters at injection to the upgrade.

Table 1

Spectrometer properties between DB5 and DB6/7.

\begin{tabular}{llllc}
\hline Beam line $\left(^{\circ}\right)$ & Quad. triplet polarities & $R_{16}(\mathbf{m})$ & $\begin{array}{l}\text { Energy resolution (\%) } \\
(\mathbf{1 ~ m m ~ e n t r y ~ s l i t ) ~}\end{array}$ & $R_{12}(\mathbf{m})$ \\
\hline 65 & $+, 0,0$ (astigmatic) & -0.53 & 0.17 & 0 \\
65 &,,+- 0 (stigmatic) & -0.86 & 0.17 & 0 \\
20 & $+, 0,0$ (astigmatic) & -0.12 & 0.50 & 0 \\
20 &,,+- 0 (stigmatic) & -0.20 & 0.50 & 0.60 \\
\hline
\end{tabular}

cavity (9GP) because of the velocity profiling of its drift tubes. The 7G3 cavity was selected instead of the 7G1 and 7G2 cavities because its geometric velocity is the closer to the beam velocity. The bulk of the measurements used the switchyard magnet to measure the energy spread of the beam as a function of the voltage of the ReB and 7G3 cavities operating in a non-accelerating mode. The energy spread was inferred from measurements of the horizontal beam size in the dispersive region after the switchyard magnet. A silicon detector under development for the longitudinal diagnostic system of the HIE-ISOLDE linac was also exploited for both energy and timing measurements. The silicon detector was placed in DB5.

The diagnostic system normally used to profile the low intensity beams at REX provides a good qualitative representation of the beam profile, however it is not well calibrated [5]. It was deemed more accurate to measure the energy spread by varying the dipole field of the switchyard magnet and scanning the beam across a slit in front of a Faraday cup located in the diagnostic boxes DB6 and DB7. A control software was custom-built for the measurement campaign in order that the beam current on the Faraday cup could be acquired as a function of the dipole field measured on a Hall probe inside the switchyard magnet.

In the absence of a dedicated offline ion source for machine development a beam consisting of residual gas from the REX EBIS was used for the measurements and a mass-to-charge state $(A / q)$ of 4 was chosen to deliver beams composed dominantly of neon buffer gas $\left({ }^{20} \mathrm{Ne}^{5+}\right)$ leaked from the adjacent Penning trap (REXTRAP). Intensities from the ion source of over 100 pA were achieved by increasing the repetition rate of the ion source close to $50 \mathrm{~Hz}$ and increasing the pressure in the EBIS to a few $10^{-10}$ mbar. As a result of leaking gas into the EBIS, which is usually done to produce high beam intensities for machine development (a few nA for all charge states), the source emittance increases as the radial potential of the electron beam trapping the ions is compensated. Nonetheless, the effect of the ion source transverse emittance on the longitudinal emittance at output from the RFQ was shown by simulation to be weak [6]. The energy spread of the beam coming from the source is estimated as $0.1 \%$ [7], which was also shown by simulation to have a negligible effect on the longitudinal emittance created in the RFQ [8].

\subsection{Spectrometer and energy spread measurements}

The switchyard magnet was incorporated into a spectrometer system comprising the diagnostic boxes and quadrupole triplet magnets located behind it on two beam lines at $20^{\circ}$ and $65^{\circ}$. The system was made point-to-point between the entry slit of the system in DB5 and the exit slits in front of the Faraday cups in DB6 and DB7 by tuning the quadrupole gradients of the triplet magnets and setting the transfer matrix elements $R_{12}$ and/or $R_{34}$ to zero (Table 1 )

$$
x_{\mathrm{DB} 6 / 7}=R_{11} x_{\mathrm{DB} 5}+R_{12} x_{\mathrm{DB} 5}^{\prime}+R_{16} \frac{\Delta p}{p} .
$$


a

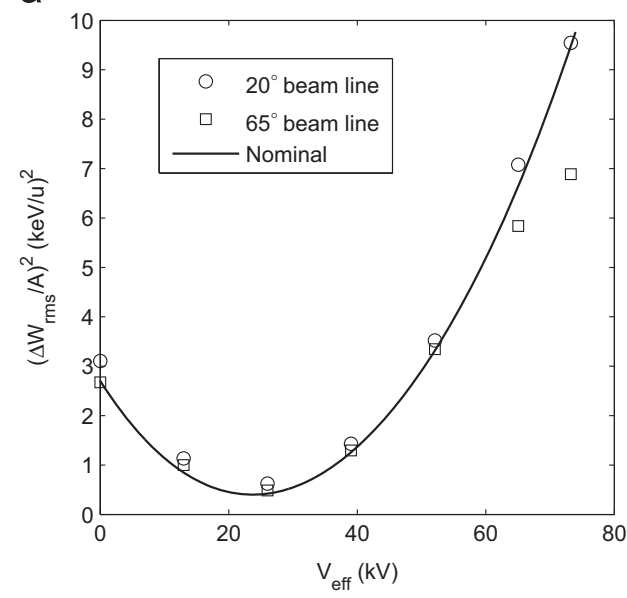

b

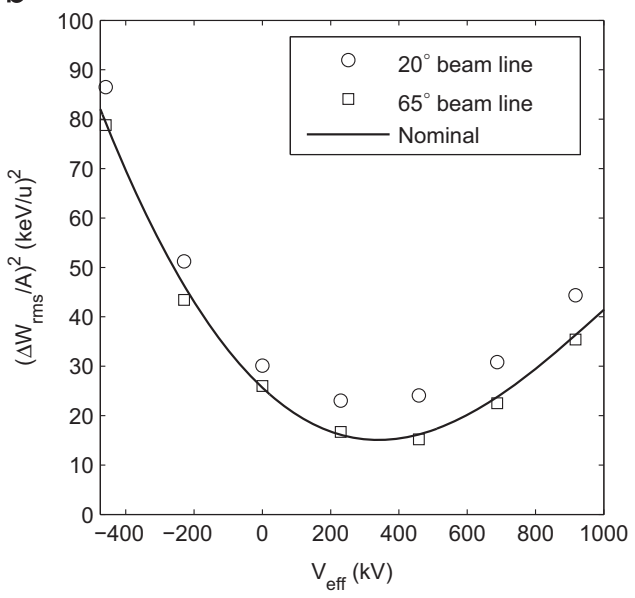

Fig. 2. TRAVEL [9] simulations: (a) Simulation of ReB measurement at $0.30 \mathrm{MeV} / u$. (b) Simulation of $7 \mathrm{G} 3$ measurement at $1.92 \mathrm{MeV} / u$.

A stigmatic focus at the Faraday cup was preferred. The dispersion function at the Faraday cup is increased when operating the system with a stigmatic focus, which reduces the perturbation of the dipole field required to move the beam across the Faraday cup. Profiling the beam by varying the dipole field of the switchyard magnet has its drawbacks because the optics of the spectrometer system is perturbed, affecting the measurement through the various listed mechanisms:

- The dispersion function changes as the radius of the beam trajectory changes.

- The edge angle seen by the beam as it leaves the switchyard magnet varies.

- The point-to-point optics solution is mismatched because the quadrupole gradients were not varied as the dipole field was scanned.

- Some of the beam may be lost on the aperture of the quadrupole as the beam is moved.

The measurement was rigorously simulated and it was shown that, depending on which beam line is used, the energy spread can be reconstructed to within a few percent, see Fig. 2 and [6]. The effect of using a circular aperture in front of the Faraday cup was shown by simulation to have a negligible effect on the measurement if a stigmatic focus was used and the vertical beam size made much smaller than the radius of the aperture. The procedure was limited by losses on the aperture of the first quadrupole after the dipole magnet. The losses were reduced with a stigmatic system but were most significant at low energy on the $65^{\circ}$ beam line as a result of the large edge angle at the exit of the switchyard magnet. The simulations indicate that losses are only problematic on the $65^{\circ}$ beam line at low energy, which is shown by the points located below the nominal energy spread at high voltages in Fig. 2a. This was indeed observed experimentally. The $65^{\circ}$ beam line was chosen for measurements at high energy because of the extra resolution required.

The beam current was very low and typically less than $10 \mathrm{pA}$ after a $1 \mathrm{~mm}$ vertical slit was placed in DB5 at the entrance to the spectrometer. Attempts were made to directly profile the beam over a slit with a size smaller than the beam in front of the Faraday cup, however in order to attain a satisfactory signal-to-noise ratio the size of the entrance slit had to be increased and the resolution of the spectrometer was compromised. This was most problematic on the more dispersive beam line where the beam size is larger and the beam intensity is spread over a wider area at the Faraday cup. Instead, the beam was accurately profiled using a slit wider than the horizontal beam size in front of the Faraday cups in DB6 and DB7. The beam profile was reconstructed from the derivative of the beam current with respect to the dipole field of the switchyard magnet as the beam was swept over each edge of the aperture, as described in Ref. [10]. The dipole field of the switchyard magnet was calibrated by assuming that the beam energy after the RFQ was $300 \mathrm{keV} / u$, which is a reasonable assumption to within the $\pm 1.5 \%$ energy spread of the beam [11]. An example profile measurement at $1.92 \mathrm{MeV} / u$ using the 7G3 is shown in Fig. 3 alongside the reconstructed profile on each side of the slit, which involved smoothing, fitting and differentiating the data.

\subsection{Energy spread measurements in the thin lens approximation}

In the thin lens approximation the linearised mapping of the longitudinal coordinates through a bunching cavity can be written

$$
\left(\begin{array}{c}
\Delta t_{1} \\
\Delta W_{1}
\end{array}\right)=\left(\begin{array}{cc}
1 & 0 \\
-q V_{\mathrm{eff}} \sin \phi_{s} & 1
\end{array}\right)\left(\begin{array}{c}
\Delta t_{0} \\
\Delta W_{0}
\end{array}\right)
$$

and expressed as

$$
X_{1}=R_{\text {buncher }} X_{0}
$$

where $\phi_{s}= \pm 90^{\circ}$ is the synchronous phase, $\Delta t$ is the bunch length, $\Delta W$ is the energy spread and $V_{\text {eff }}$ is the effective voltage including the transit time factor. In the sigma matrix formalism

$$
\sigma=\left(\begin{array}{cc}
\Delta t^{2} & \Delta t \Delta W \\
\Delta W \Delta t & \Delta W^{2}
\end{array}\right)=\epsilon\left(\begin{array}{cc}
\beta & -\alpha \\
-\alpha & \gamma
\end{array}\right)
$$


a

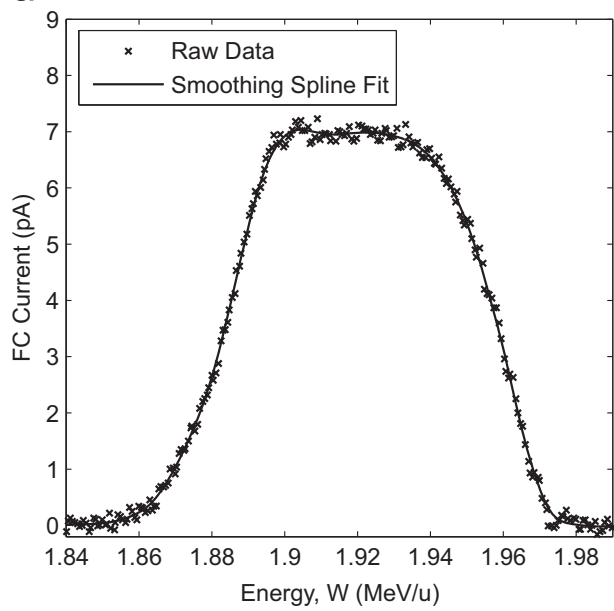

b

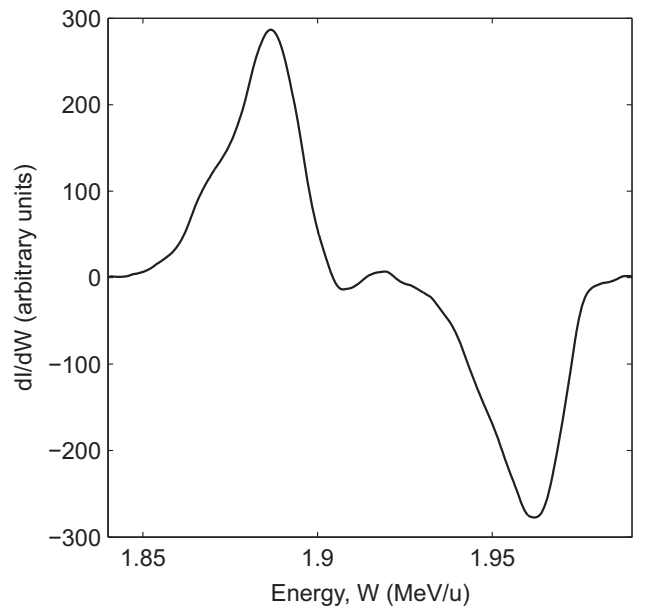

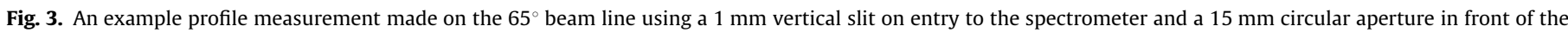
Faraday cup: (a) Raw data with smoothed fit. (b) Derivative of the fit.

the Twiss parameters $(\alpha, \beta, \gamma$ and $\epsilon)$ can be tracked through a transfer matrix $(R)$ by calculating

$$
\sigma_{1}=R \sigma_{0} R^{T}
$$

The square of the energy spread measured after the bunching cavity $\left(\Delta W_{1}^{2}\right)$ can be written as a quadratic function of the effective voltage and the Twiss parameters immediately before the cavity

$$
\frac{\Delta W_{1}^{2}}{A^{2}}=\epsilon_{0}\left[\left(\frac{q}{A}\right)^{2} \beta_{0} \sin ^{2} \phi_{s} V_{\text {eff }}^{2}+2\left(\frac{q}{A}\right) \alpha_{0} \sin \phi_{s} V_{\text {eff }}+\gamma_{0}\right]
$$

where the mass number $(A)$ of the ion is included explicitly to show the effect of the mass-to-charge state on the measurement. Experimentally the emittance can be determined from a quadratic fit of the form $y=a_{2} x^{2}+a_{1} x+a_{0}$, where $x=V_{\text {eff }}$ sin $\phi_{s}$ and $y=\Delta W_{1}^{2} / A^{2}$. The emittance can be expressed,

$$
\epsilon_{0}=\left(\frac{A}{q}\right) \sqrt{a_{0} a_{2}-\frac{a_{1}^{2}}{4}}
$$

and the other Twiss parameters can be written

$$
\begin{aligned}
& \beta_{0}=\left(\frac{A}{q}\right)^{2} \frac{a_{2}}{\epsilon_{0}} \\
& \alpha_{0}=\frac{1}{2}\left(\frac{A}{q}\right) \frac{a_{1}}{\epsilon_{0}}
\end{aligned}
$$

and

$$
\gamma_{0}=\frac{a_{0}}{\epsilon_{0}}
$$

\subsection{Energy spread measurements with multi-gap bunching cavities}

The ReB and 7G3 cavities have 3 and 7 accelerating gaps respectively, which limits the application of the thin lens approximation in the three-gradient emittance measurement. The extent of the limitation was investigated and a formalism was developed to extend the thin lens approximation to make accurate emittance measurements with multi-gap cavities. The longitudinal transfer matrix $R_{\text {buncher }}^{N \text { gaps }}$ describing the dynamics between the first and last gaps of an $\mathrm{N}$-gap bunching cavity with a constant geometric velocity operating in $\pi$-mode can be calculated by concatenating the matrices

$$
R_{\text {buncher }}^{N \text { gaps }}=R_{1 \text {, buncher }} R_{1 \text {,drift }} R_{2 \text {,buncher }} R_{2 \text {,drift }}, \ldots, R_{N-1 \text {, buncher }} R_{N-1 \text {,drift }} R_{N \text {, buncher }}
$$

where the $i$ th gap is approximated by a thin element. The drift distances in a $\pi$-mode structure with constant gap spacing can be mapped as

$$
R_{i+1, \text { drift }}=R_{i, \text { drift }}=\left(\begin{array}{cc}
1 & -\frac{\pi}{2 W_{0}} \\
0 & 1
\end{array}\right)
$$

where the average beam energy $\left(W_{0}\right)$ is assumed to be matched to the geometric velocity of the cavity. The total effective bunching voltage is defined by the sum of the voltages in each gap $\left(V_{i, \text { eff }}\right)$ such that,

$$
V_{\text {eff }}=\sum_{i=1}^{N} V_{i, \text { eff }}
$$


The matched beam velocity remains constant between the gaps at the non-accelerating phases and the synchronous phase is the same in each gap. After expanding out Eq. (11) one can write

$$
R_{\text {buncher }}^{N \text { gaps }}=\sum_{i=1}^{N}\left(\begin{array}{cc}
f_{i, 11}(N)\left(\frac{\pi}{2} \sin \phi_{s}\right)^{i-1}\left(\frac{q V_{\text {eff }}}{W_{0}}\right)^{i-1} & -\frac{\pi}{2 W_{0}} f_{i, 12}(N)\left(\frac{\pi}{2} \sin \phi_{s}\right)^{i-2}\left(\frac{q V_{\text {eff }}}{W_{0}}\right)^{i-2} \\
-q V_{\text {eff }} \sin \phi_{s} f_{i, 21}(N)\left(\frac{\pi}{2} \sin \phi_{s}\right)^{i-1}\left(\frac{q V_{\text {eff }}}{W_{0}}\right)^{i-1} & f_{i, 22}(N)\left(\frac{\pi}{2} \sin \phi_{s}\right)^{i-1}\left(\frac{q V_{\text {eff }}}{W_{0}}\right)^{i-1}
\end{array}\right) .
$$

The matrix elements of $R_{\text {buncher }}^{N \text { gaps }}$ are finite polynomial expansions in $q V_{\text {eff }} / W_{0}$ with an order less than $N$ and with coefficients $f_{i, j k}$ that are simple functions of $N$. Each term in the expansion can be written in matrix form

$$
R_{\text {buncher }}^{N \text { gaps }}=\sum_{i=1}^{N} R_{i}
$$

In this form one can truncate the polynomials and write approximate expressions for the transfer matrix when $q V_{\text {eff }} \ll W_{0}$. The transfer matrix for an $\mathrm{N}$-gap bunching cavity can be approximated as

$$
R_{\text {buncher }}^{N \text { gaps }} \approx R_{1}+R_{2}+R_{3}
$$

where $R_{1}$ represents the thin lens approximation. The next two matrices act as corrections, sufficient to calculate the beam parameters downstream to second-order in $q V_{\text {eff }} / W_{0}$. The coefficients $f_{i, j k}$ depend on the voltage distribution across the gaps inside the cavity and were derived up to $i=3$ for a flat voltage distribution and a flat distribution with the external drift tubes grounded [6]. By truncating the expansion at $i=2$, such that $R_{\text {buncher }}^{N}=\sum_{i=1}^{2} R_{i}=R_{1}+R_{2}$, the energy spread downstream of a multi-gap bunching cavity can be approximated to orders linear in $q V_{\text {eff }} / W_{0}$ as a cubic function of $V_{\text {eff }}$

$$
\left(\frac{\Delta W_{1}^{2}}{A^{2}}\right)=a_{3} \sin ^{3} \phi_{s} V_{\text {eff }}^{3}+a_{2} \sin ^{2} \phi_{s} V_{\text {eff }}^{2}+a_{1} \sin \phi_{s} V_{\text {eff }}+a_{0}
$$

where

$$
\begin{aligned}
& \frac{a_{3}}{\epsilon_{0}}=\left(\frac{q}{A}\right)^{3} \frac{\pi}{W_{0} / A} f_{2,21} \beta_{0} \\
& \frac{a_{2}}{\epsilon_{0}}=\left(\frac{q}{A}\right)^{2}\left[\beta_{0}+\frac{\pi}{W_{0} / A}\left(f_{2,21}+f_{2,22}\right) \alpha_{0}\right] \\
& \frac{a_{1}}{\epsilon_{0}}=\left(\frac{q}{A}\right)\left[2 \alpha_{0}+\frac{\pi}{W_{0} / A} f_{2,22} \gamma_{0}\right] \\
& \frac{a_{0}}{\epsilon_{0}}=\gamma_{0} .
\end{aligned}
$$

By truncating the expansion at $i=3$ the energy spread can be written as a quartic function and similar relationships between the fit coefficients and the Twiss parameters can be derived. The coefficients of the fitted polynomials in these extensions of the thin gap approximation characterise more simultaneous equations than independent and unknown Twiss parameters. The problem is overconstrained and a unique expression for the emittance does not exist as it does for the quadratic case. Nonetheless, one can implement the above relationships into a least-square fitting routine to solve for the beam parameters from experimental data. The energy spread of the beam calculated downstream of the ReB and 7G3 cavities is plotted using nominal beam parameters taken from simulation as a function of typical values of $q V_{\text {eff }} / W_{0}$ in Fig. 4. The $V_{\text {eff }}$ dependence of the complete transfer matrix $R_{\text {buncher }}^{N \text { gap }}$ is compared with the simple thin lens approximation
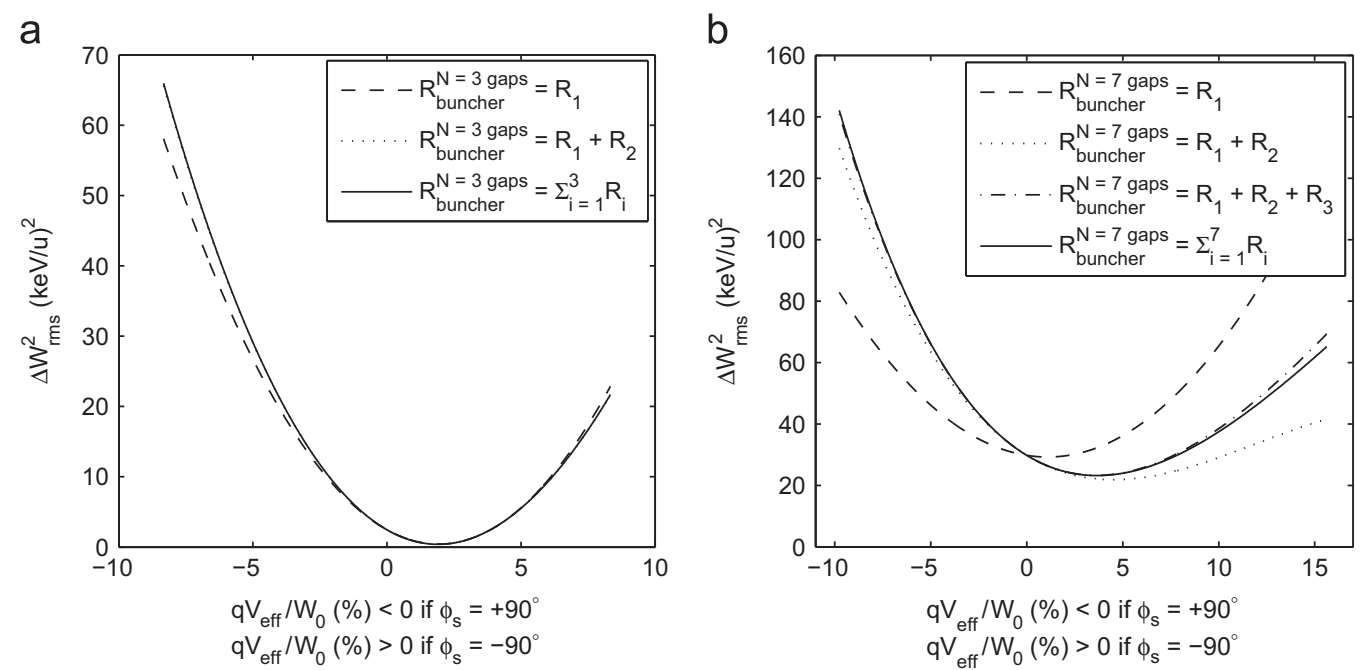

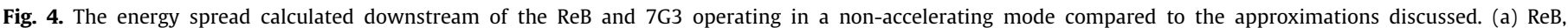
$W_{0}=300 \mathrm{keV} / u$. (b) $7 \mathrm{G} 3, W_{0}=1.92 \mathrm{MeV} / u$. 
and the extensions described above. Although the thin lens approximation is reasonable for the ReB, it is imperative that the thin lens approximation is extended in the case of the 7G3.

\section{ReB measurements}

Emittance measurements were made at various RFQ voltages and the nominal beam parameters at the RFQ exit were found close to a signature minimum in the energy spread, which correlates well with simulation [8]. The ReB was not stable when operated below an amplitude corresponding to an effective voltage of $35 \mathrm{kV}$ and the energy spread was too large to take measurements at the debunching synchronous phase of $+90^{\circ}$. Although initial measurements were made on the $65^{\circ}$ beam line of the spectrometer, it was challenging to ensure transmission through the spectrometer as the dipole field was perturbed and the $20^{\circ}$ beam line was preferred instead.

\subsection{Spectrometer measurements}

The emittance was inferred from a quadratic fit to the data plotted in Fig. 5 using Eq. (6). The fit, which was weighted with the random errors shown by the error bars in Fig. 5, gave an rms emittance of $0.34 \pm 0.08 \pi \mathrm{ns} \mathrm{keV/u}$ with a $1 \mathrm{~mm}$ entry slit. An estimate of the systematic error arising from the contribution to the resolution of the finite width of the entry slit was made by comparing the measured beam profiles with different slit sizes, as is shown explicitly in Fig. 6 for the $7 \mathrm{G} 3$ measurement. The resolution from the $1 \mathrm{~mm}$ slit was estimated at $0.4 \pm 0.1 \mathrm{keV} / u$. This corresponds to a resolution of $0.13 \%$, which is considerably lower than the estimated resolution of $0.50 \%$. After subtraction of the resolution the emittance is estimated at $0.29 \pm 0.07 \pi \mathrm{ns} \mathrm{keV/u}$. The measured beam parameters, which are summarised in Table 2 , are affected by the

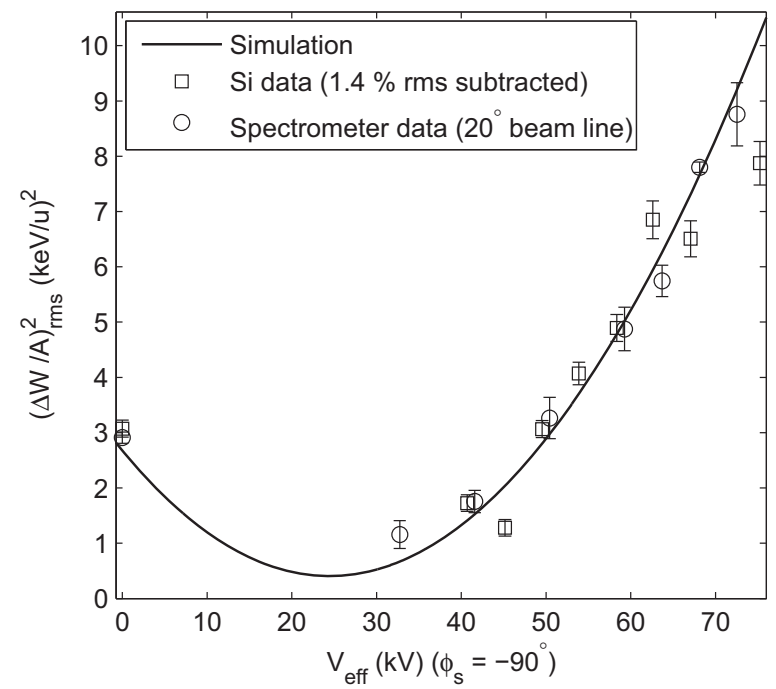

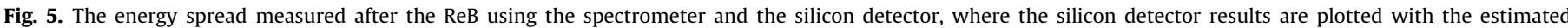
resolution subtracted.

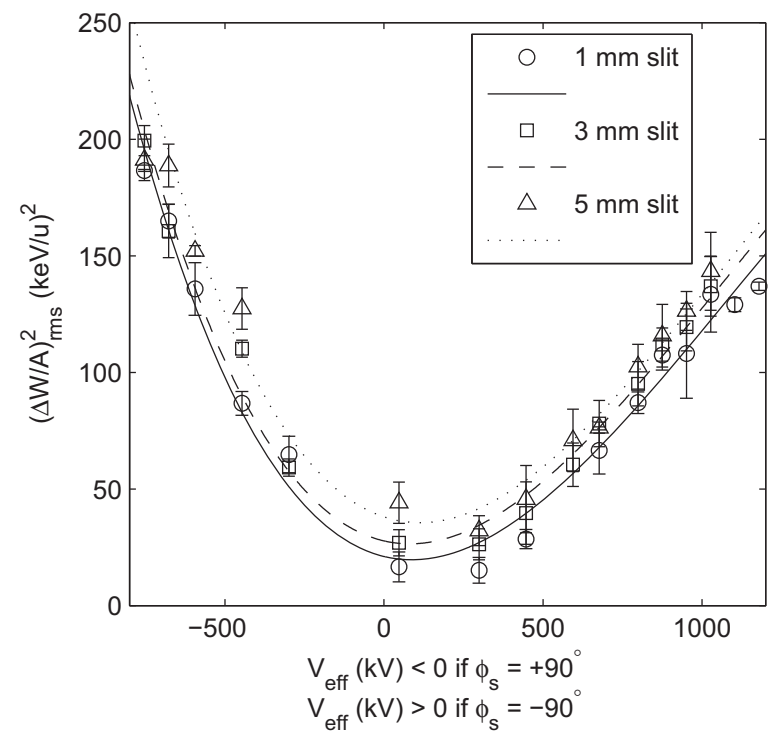

Fig. 6. Emittance measurement with the 7G3 using the spectrometer with different entry slit sizes. 
resolution but are still in good agreement with simulation. The voltage required to minimise the energy spread, which is independent of the resolution and proportional to $\alpha_{0} / \beta_{0}$, is consistent with simulation.

\subsection{Silicon detector measurements}

In accordance with an investigation of the energy resolution of the solid-state diagnostic system the longitudinal emittance was also measured. The rms emittance measured with the silicon detector is a factor of 4.6 times larger than the measurement with the spectrometer on the $20^{\circ}$ beam line and a factor of 6 times larger than simulation. The system performs well in comparison to other solidstate systems used to measure the longitudinal emittance [12,13] but the estimated resolution of $1.4 \%$ rms still severely impaired the measurement, as shown by the measured beam parameters collected in Table 2. The limited energy resolution makes accurate measurements of the beam energy spread challenging with the solid-state system. After the subtraction of the resolution in quadrature, the data agrees closely with both the spectrometer measurement and simulation, as shown in Fig. 5. The value of the resolution independent ratio of $\alpha_{0} / \beta_{0}$ is in close agreement with simulation and the spectrometer measurement. Further details of the measurements with the solid-state diagnostic system can be found in Ref. [14].

\section{7G3 measurements}

In nominal operation the beam has an energy of $1.9 \mathrm{MeV} / u$ at the $7 \mathrm{G} 3$ and a reduced velocity of $6.4 \%$, which is lower than the geometric velocity of the 7G3 cavity of $6.6 \%$ [15]. Consequently the phase deviates from the non-accelerating phases of $\pm 90^{\circ}$ in each gap. The variation was shown to be at most $\pm 20^{\circ}$ in the first and last gaps where the voltage is lower than that in the other gaps because the external drift tubes are grounded. The effect of the variation of the synchronous phase in each gap was shown by simulation to be small and even with the mismatched velocity the emittance could be reconstructed to within $12 \%$ [6].

\subsection{Spectrometer measurements}

The energy spread was measured on the $65^{\circ}$ beam line as a function of the effective voltage of the $7 \mathrm{G} 3$ as presented in Fig. 6 with three different vertical slits placed at entry to the spectrometer to illustrate the effect of the entry slit size on the resolution of the measurement. The energy spread was reconstructed from scans across a $15 \mathrm{~mm}$ circular aperture in front of the Faraday cup. The error bars on each data point in Fig. 6 reflect the standard deviation of six measurements of the energy spread taken from three spectrometer scans made at each voltage. The non-quadratic behaviour of the 7G3 is evident through the asymmetry of the data about the minimum and quartic functions were fitted to the data, giving emittances of $0.36 \pm 0.05,0.42 \pm 0.04$ and $0.51 \pm 0.02 \pi \mathrm{ns} \mathrm{keV} / \mathrm{u}$ for 1,3 and $5 \mathrm{~mm}$ entry slits, respectively. At low voltages the resolution contribution from a $1 \mathrm{~mm}$ slit is estimated as $1.0 \pm 0.2 \mathrm{keV} / u$, which is negligible when compared to the random error involved in the measurement. This corresponds to a resolution of $0.05 \%$, considerably lower than the estimated resolution of $0.17 \%$ but a similar factor lower than that was estimated for the ReB measurement on the $20{ }^{\circ}$ beam line. The rms emittance is measured by a factor of 1.125 larger than simulation and as is summarised in Table 3.

\section{Longitudinal phase space distribution}

The distribution of the energy spread profiles were analysed and the beam parameters for phase space ellipses containing different fractions of the beam were calculated by applying the three-gradient method in the same way as was described above for the rms emittance. The ellipses were then interpolated in the longitudinal phase plane, as is shown in Fig. 7 at a position directly in front of the $\mathrm{ReB}$, where the measured contours are compared to the simulated particle distribution. This indirect measurement of the distribution compares well with simulation, as summarised in Fig. 8, although the sub-structure predicted by simulation is not resolvable. The signalto-noise ratio allowed the distribution to be probed reliably up to an emittance containing $86 \%$ of the beam. The core of the beam is closely Gaussian but particles further from the core populate more extreme regions of phase space than that is described by the Gaussian distribution; low energy tails were observed [6].

\section{Summary tables}

The results of the measurement campaign are summarised in Tables 2 and 3.

Table 2

Summary table of the longitudinal emittance measurements with $\mathrm{ReB}$ at $300 \mathrm{keV} / \mathrm{u}^{\mathrm{a}}$

\begin{tabular}{|c|c|c|c|c|c|}
\hline Measurement & $\alpha$ & $\beta(\mathbf{n s} / \mathbf{k e V} / \mathbf{u})$ & $\alpha / \beta(\mathbf{n s} / \mathbf{k e V} / \boldsymbol{u})$ & $\epsilon_{\mathrm{rms}}(\boldsymbol{\pi} \mathbf{n s} \mathbf{k e V} / \boldsymbol{u})$ & $\epsilon_{86 \%}(\pi$ ns keV $/ \mathbf{u})$ \\
\hline Spectrometer $\left(20^{\circ}\right)$ & 1.58 & 0.41 & 3.85 & $0.34 \pm 0.08$ & $1.48 \pm 0.2$ \\
\hline Si detector & 0.29 & 0.074 & 3.92 & $1.56 \pm 0.17$ & $5.44 \pm 0.7$ \\
\hline Simulation & 2.25 & 0.60 & 3.75 & 0.26 & 0.96 \\
\hline
\end{tabular}

a Beam parameters given in front of the ReB without any correction for the resolution. 
Table 3

Summary table of the longitudinal emittance measurements with $7 \mathrm{G} 3$ at $1.92 \mathrm{MeV} / \mathrm{u}^{\mathrm{b}}$

\begin{tabular}{lllll}
\hline Measurement & $\alpha$ & $\beta(\mathbf{n s} / \mathbf{k e V} / \boldsymbol{u})$ & $\epsilon_{\text {rms }}(\boldsymbol{\pi} \mathbf{n s ~ k e V / u})$ & $\epsilon_{86 \%}(\boldsymbol{\pi} \mathbf{n s ~ k e V} / \boldsymbol{u})$ \\
\hline Spectrometer $\left(65^{\circ}\right)$ & 0.06 & 0.02 & $0.36 \pm 0.05$ & $1.55 \pm 0.12$ \\
Simulation & 0.05 & 0.008 & 0.32 & 1.03 \\
\hline
\end{tabular}

${ }^{\mathrm{b}}$ Beam parameters given in front of the 7G3 without any correction for the resolution.

a

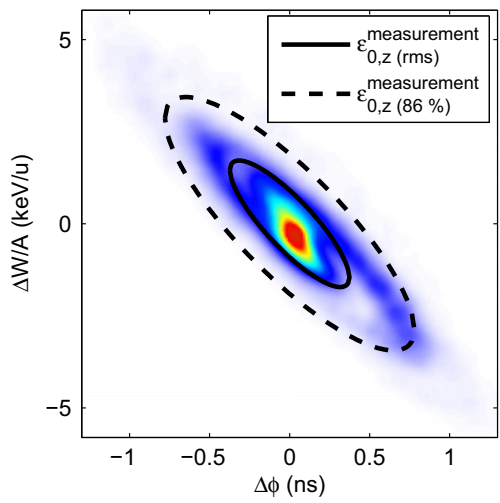

b

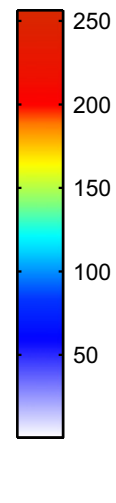

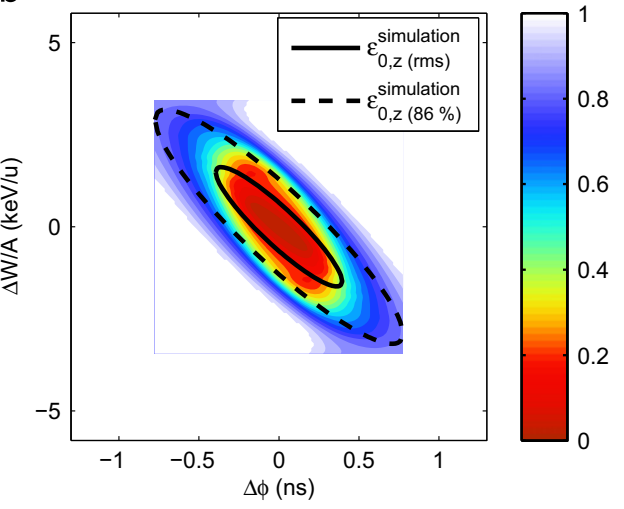

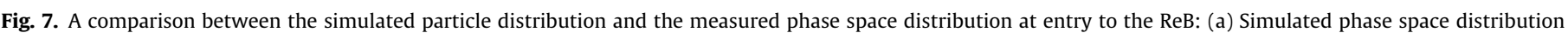

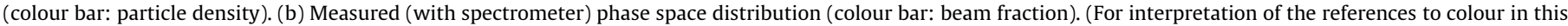
figure legend, the reader is referred to the web version of this article.)

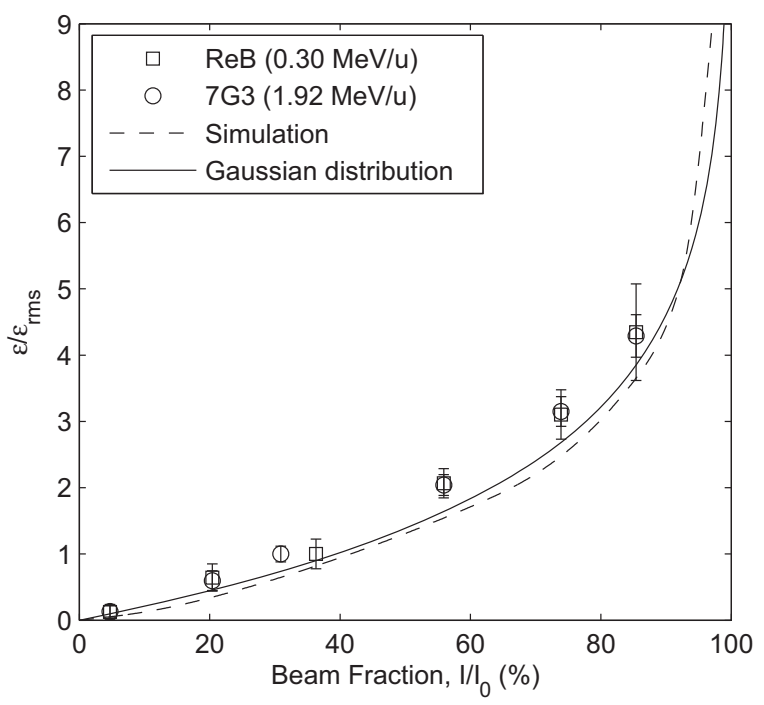

Fig. 8. An indirect measurement of the distribution of the beam in longitudinal phase space.

\section{Conclusion}

The rms longitudinal emittance at output from the RFQ was measured as $0.34 \pm 0.08 \pi \mathrm{ns} \mathrm{keV} / u$ and at entry to the 7G3 as $0.36 \pm 0.04 \pi \mathrm{ns} \mathrm{keV} / u$, indicating a small growth of emittance in the IHS. After the subtraction of the estimated resolution from the $1 \mathrm{~mm}$ slit in front of the spectrometer the rms emittance measured before the IHS is $0.29 \pm 0.07 \pi \mathrm{ns} \mathrm{keV} / u$, which gives an emittance growth in the IHS, 7G1 and 7G2 cavities of approximately $20 \%$, consistent with simulation. The $86 \%$ emittance was measured by a factor of approximately 4.4 times larger than the rms emittance at $1.48 \pm 0.2$ and $1.55 \pm 0.12 \pi \mathrm{ns} \mathrm{keV} / u$ at the RFQ and 7G3, respectively. Systematic errors arising from the resolution of the spectrometer and the mismatch of the beam velocity with the bunching cavity's geometric velocity were estimated at approximately $10 \%$. A silicon detector in its development phase was also exploited to measure the longitudinal beam properties but the measurements were resolution limited.

An emittance after the RFQ of $2 \pi \mathrm{ns} \mathrm{keV/u}$ is consistent with simulation and the recent measurement campaign. The emittance can be accepted into the longitudinal acceptance of the superconducting HIE-ISOLDE linac, as shown in Fig. 9 for two stages of the upgrade; at injection to the upgrade after the IHS and 9GP structures. Simulations show that the 9GP cavity increases the rms longitudinal emittance by approximately a factor of 2 . The realistic particle distribution at the RFQ output was tracked through the REX linac up to the first cavity of the HIE linac in stages 1 and $2 b$ of the upgrade, without and with the low energy section, respectively. 
a

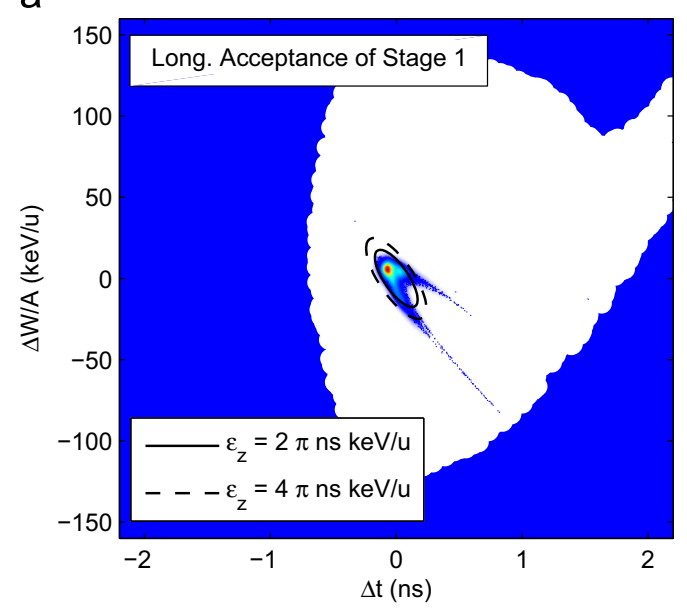

b

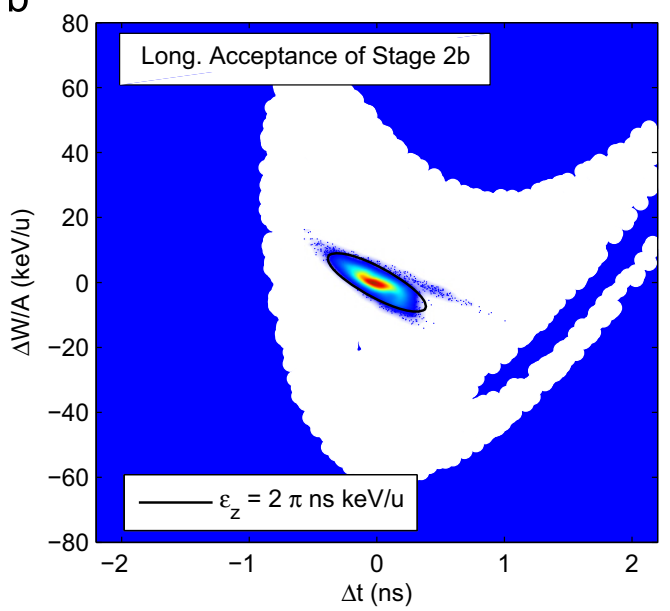

Fig. 9. The REX beam compared to the longitudinal acceptance of the HIE-ISOLDE linac: (a) Stage $1, W_{0}=2.8 \mathrm{MeV} / u$. (b) Stage $2 \mathrm{~b}, W_{0}=1.2 \mathrm{MeV} / u$.

\section{Acknowledgments}

The authors would like to thank E. Piselli for the preparation of the energy scanning application. M.A. Fraser acknowledges the receipt of funding from the ISOLDE Collaboration Committee and the Cockcroft Institute. F. Zocca also acknowledges the receipt of funding from the ISOLDE Collaboration Committee and from the Centro Nacional de F'sica de Part'culas, Astropart'culas y Nuclear (CPAN), Spain, project n. CSD2007-00042.

\section{References}

[1] F. Ames, J. Cederkäll, T. Sieber, F.J.C. Wenander, The REX-ISOLDE Facility: Design and Commissioning Report, CERN, Geneva, 2005.

[2] M. Pasini, et al., HIE-ISOLDE: the superconducting rib linac at CERN, in: Proceedings of SRF09, Berlin, Germany, pp. 924-929.

[3] D. Voulot, REX-linac emittance measurements, Internal Note CERN-BE-Note-2009-012, CERN, Geneva, CH, 2009.

[4] P. Strehl, Beam Instrumentation and Diagnostics, Particle Acceleration and Detection, Springer, Berlin, 2006.

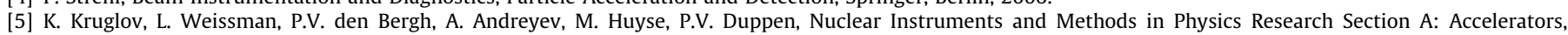
Spectrometers, Detectors and Associated Equipment 441 (2000) 595.

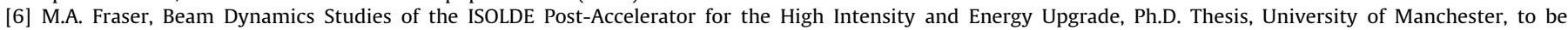
published.

[7] F. Wenander, Private communication, 2011.

[8] M.A. Fraser, P.A. Posocco, M. Pasini, Benchmarking of the REX-ISOLDE RFQ Beam Dynamics Simulations, BE Dept. Note, CERN, 2011.

[9] A. Perrin, J. Amand, T. Mütze, Travel v4.07 User Manual, CERN, 2007.

[10] H. Wollnik, Optics of Charged Particles, Academic Press, Orlando, FL, 1987.

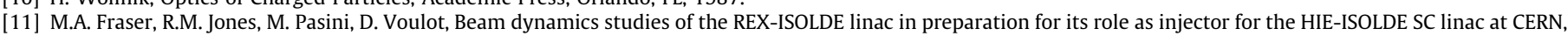
in: Proceedings of LINAC10, Tsukuba, Japan, pp. 950-952.

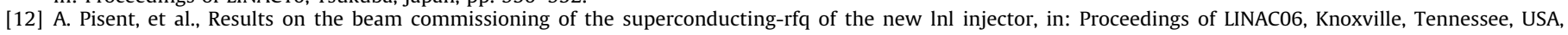
pp. 227-229.

[13] T. Milosic, P. Forck, Longitudinal emittance measurement using particle detectors, in: Proceedings of DIPAC09, Basel, Switzerland, pp. 330-332.

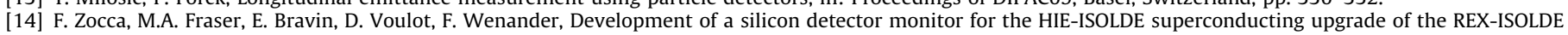
heavy-ion linac, in: Proceedings of DIPAC11, Hamburg, Germany.

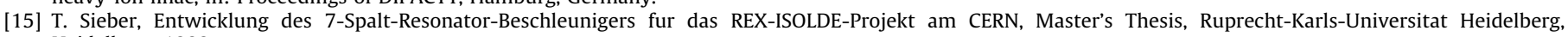
Heidelberg, 1999. 